
\title{
CR Research Square \\ The Roles of Matrix Metalloproteinase 7 in Spinal Cord Ischemia- Reperfusion Injury in Rats
}

\section{Te Fang}

The First Hospital of China Medical University: The First Affiliated Hospital of China Medical University

\section{Fengshou Chen}

The First Affiliated Hospital of China Medical University: The First Hospital of China Medical University

\section{Dan Wang}

The First Hospital of China Medical University: The First Affiliated Hospital of China Medical University

\section{Zaili Zhang ( $\nabla$ zhangzaili333@sina.com )}

The First Affiliated Hospital of China Medical University: The First Hospital of China Medical University https://orcid.org/0000-0001-7897-8680

\section{Research}

Keywords: MMP-7, spinal cord ischemia-reperfusion injury, neuroapoptosis, inflammation

Posted Date: November 10th, 2021

DOI: https://doi.org/10.21203/rs.3.rs-1023579/v1

License: (c) (1) This work is licensed under a Creative Commons Attribution 4.0 International License. Read Full License 


\section{Abstract}

Background: Neuronal survival after spinal cord ischemia-reperfusion injury (SCII) is a major factor affecting the motor function. Recently, matrix metalloproteinase-7 (MMP-7) plays an important role in a variety of diseases, but the specific role of MMP-7 in SCII remains unclear. This paper aims to further investigate the role of MMP7 in SCII.

Methods: We built the SCII model by clipping the aortic arch for 14 minutes. The RNA and protein expression of MMP-7 was detected by Western blot and real-time polymerase chain reaction (PCR) during the groups. The co-localization of major cell types and MMP-7 in the spinal cords was detected by Immunofluorescence staining. To further study the specific mechanism, rats were intrathecally pretreated with si-MMP7 or negative control (NC) siRNA 3 days before clipping the aortic arch for 14 minutes. The Tarlov criteria and Haematoxylin and eosin staining were used to detect neurological function and histological assessment. Western blot, PCR and Immunofluorescence staining were used to evaluate the effect of silencing MMP-7.

Results: The expression of MMP-7 RNA and protein increased both at $12 \mathrm{~h}$ and $24 \mathrm{~h}$ after SCII. At $24 \mathrm{~h}$ after SCII, the expression of MMP-7 RNA reached its maximum amount. Compared with the sham group, MMP-7 fluorescent expression in the SCIl group greatly enhanced, and MMP-7 fluorescence was mainly co-expressed with neuronal nuclei (NeuN) fluorescence. The Tarlov scores and number of intact neurons were increased by pre-treatment with si-MMP7. Pre-treatment with si-MMP7 could also decrease the expression of MMP-7, Interleukin-1 $\beta$ (IL-1 $\beta$ ) and cleaved caspase-3 and reduce the MMP-7 expression in neurons.

Conclusions: Silencing MMP-7 protected the rats against SCII by inhibiting the neuroapoptosis and inflammation. Silencing MMP-7 could be a hopeful therapeutic treatment after SCII.

\section{Background}

Spinal cord ischemia-reperfusion injury (SCII) is a devastating and unpredictable complication that can lead to paraplegia after aortic aneurysm repair [1]. The complication is still not completely preventable with the development of surgical techniques and medical interventions [2,3]. Motor dysfunction is due to the massive neuronal death after ischemic-reperfusion injury, which is thought to be one of the mechanisms of SCII [4-6]. Thus, decreasing neuronal death may represent a hopeful strategy for relieving motor dysfunction after SCII.

Matrix metalloproteinases (MMPs) are zinc-containing endopeptidases that can cleave and degrade the extracellular matrix [7]. MMPs are functional classified as gelatinases (MMP2 and MMP9), collagenases (MMP1, MMP8 andMMP13), matrilysin (MMP7 and MMP26), and stromelysins (MMP3, MMP10 and MMP11) [8]. MMP-7 is one of the smallest MMPs and a secreted zinc-dependent endopeptidase [9, 10]. MMP-7 plays a vital role in the modulation of multiple biological processes. For example, MMP-7 is upregulated in several kinds of cancer [11, 12]; and MMP-7 protects against acute kidney injury by 
priming renal tubules for survival and regeneration [13]. In neurological diseases, MMP-7 is greatly increased in rat sciatic nerve segments after nerve injury [14]; and MMP-7 is on the regulation of Schwann cell phenotype and the regeneration of injured peripheral nerves [15]; and MMP-7 removes remyelinationimpairing fibronectin aggregates in multiple sclerosis lesions [16]. Additionally, it has been confirmed in our previous study that MMP-7 was involved in SCII [17], but the mechanism has not been fully understood. In this study, we explore the specific role of MMP-7 in the pathogenesis of SCII to provide a theoretical basis for finding effective therapies.

\section{Material And Methods \\ 2.1. Animals and regents}

All male Sprague-Dawley (SD) rats ( $n=72)$ weighing about 220-260 g were purchased from the Animal Center of China Medical University. The protocols of animal experiments were approved by the Ethics Review Committee of China Medical University. The rats were housed in standard cage under a temperature-controlled $\left(22-24^{\circ} \mathrm{C}\right)$ condition, with free food and water.

MMP-7 small interfering RNAs (siRNAs) were provided by Shanghai GenePharma Company. MMP-7 siRNA sequence (5'-CCUACAGAAUCGUGUCCUATT-3', 5'-UAGGACACGAUUCUGUAGGTT-3') and Scramble siRNA (si-NC) sequence (5'-UUCUCCGAACGUGUCACGUTT-3', 5'-ACGUGACACGUUCGGAGAATT-3') were designed.

\subsection{SCII model}

The SCII model was finished, as described previously [18]. Animals were injected intraperitoneally with $4 \%$ sodium pentobarbital ( $50 \mathrm{mg} / \mathrm{kg}$; Beyotime Biotechnology, Shanghai, China). The distal and proximal blood pressure was calculated by two catheters which were respectively inserted into the tail artery or the left carotid artery. After endotracheal intubation and mechanical ventilation, the aortic arch of rats was revealed by a left thoracotomy. In a direct visualization, the aortic arch was occluded between the left carotid artery and left subclavian artery to cause spinal cord ischemia.

Useful ischemia was kept for 14 minute, which was verified by observing a $90 \%$ reduction in flow measured at the tail artery. In the Sham group, rats were subjected to the same process without occluding the aortic arch.

\subsection{Intrathecal infusion of siRNAs}

The injection space was located between segments $L_{4}$ and $L_{6}$ in the dura. Phenomenon of tail flick was the sign confirming the correct position [19]. According to the preliminary experiment, $25 \mu \mathrm{l}$ of si-MMP7 or NC was intrathecally injected with Lipofectamine 3000 (Invitrogen, USA) at a concentration of $2.5 \mu \mathrm{g} / \mu \mathrm{l}$. After 3 intrathecal injections at $24 \mathrm{~h}$ intervals, rats with no evidence of motor function deficit underwent surgery.

\subsection{Behavioral assessment}


The behavioral assessment of the rat's hind limb was measured with Tarlov criteria after SCIl by two watchers who were unfamiliar with the experiment. Tarlov scores were ranged from 0 (no hind limb motor function) to 4 (normal) in previous study [20].

\subsection{Experimental protocol}

In Figure.1A-B, the experimental protocol was shown. The rats $(n=72)$ were divided into four or five groups using a random number table. As shown in Fig. 1A, the expression of MMP-7 was measured at different time points (12h, $24 \mathrm{~h}, 36 \mathrm{~h}$, and $48 \mathrm{~h}$ ) after SCII. Then, the rats were randomly arranged to four groups at $24 \mathrm{~h}$ after SCll in the Fig. 1B: (1) sham group: rats were performed $25 \mu \mathrm{l}$ of saline by intrathecal injection before sham-operation at $24 \mathrm{~h}$ intervals for three consecutive days. (2) SCll group: rats were performed 25 $\mu \mathrm{l}$ of saline by intrathecal injection before SCIl-operation at $24 \mathrm{~h}$ intervals for three consecutive days. (3) SCll+negative control (NC) group: $25 \mu \mathrm{l}$ of NC was intrathecally injected into rats before SCIl-operation at $24 \mathrm{~h}$ intervals for three consecutive days. (4) SCII+si-MMP7 group: $25 \mu \mathrm{l}$ of si-MMP7 was intrathecally injected into rats before SCII-operation at $24 \mathrm{~h}$ intervals for three consecutive days.

\subsection{Quantitative real-time polymerase chain reaction (PCR)}

The TRIzol reagent was used to extract the total RNA from $L_{4-6}$ sections of spinal cords. The PrimeScript RT reagent kit (RR037A Takara) was used to reversely transcribe the cDNA. Then, a TB Green Premix Ex Taqll kit (Takara) on a Prism 7000 detection system (Takara) was used to analyze the cDNA. GAPDH was used to normalize the relative MMP-7 expression, and all samples were measured in triplicate by the $2^{-\triangle \triangle C T}$ method. The primers used in the study were as follows: MMP-7 (5'- CACGGAGAC AACTTCCCATT3', 5'- CACCATCCGTCCAGTACTCA-3'), and GAPDH (5'- GGGGCTCTCTGCTCCTCCCTG-3', 5'AGGCGTCCGATACGGCCAAA -3').

\subsection{Western blot}

$\mathrm{L}_{4-6}$ spinal cord segments were homogenized and collected at $4^{\circ} \mathrm{C}$. After extraction with a BCA protein kit (Beyotime Biotechnology), the protein samples were loaded onto $8 \%$ sodium dodecylsulfate polyacrylamide gel electrophoresis and then transferred to polyvinylidene difluoride membranes. The membranes were blocked with $5 \%$ skim milk for $1 \mathrm{~h}$ and were incubated overnight at $4^{\circ} \mathrm{C}$ with primary antibodies against IL-1 $\beta$ (Abcam, ab9722, 1:2000), cleaved caspase-3 (Affinity, AF7022, 1:500), MMP-7 (Affinity, AF0218, 1:500), and $\beta$-actin (Abcam, ab8227, 1:10000). The membranes were washed with TBST (Solarbio) and incubated with relevant secondary antibodies (Beyotime, 1: 1000). The bands were detected by an ECL kit (Bio-Rad, USA) and software (Bio-Rad Laboratories).

\subsection{Immunofluorescence (IF) staining}

The cellular distribution of MMP-7 expression was measured by immunofluorescence staining at the $24 \mathrm{~h}$ time point after SCII. Spinal cord segments were fixed and cut into $8 \mu \mathrm{m}$ sections. The segments were sealed with $10 \%$ bovine serum albumin (BSA) and incubated with primary antibodies overnight at $4^{\circ} \mathrm{C}$. Then, they were incubated with the corresponding secondary antibodies for $2 \mathrm{~h}$ at room temperature. Primary antibodies included mouse anti-NeuN (Abcam, ab104224, 1:400), goat anti-GFAP (Abcam, 
ab53554, 1:400), mouse anti-Iba-1 (Abcam, ab5076, 1:400), rabbit anti-MMP-7 (Affinity, AF0218, 1:200) and mouse anti-cleaved caspase-3 (Abmart, M020539, 1:50). Corresponding secondary antibodies included FITC-conjugated AffiniPure donkey anti-goat IgG $(H+L)$ (Proteintech, 1:200), Alexa Fluor 594conjugated donkey anti-rabbit lgG $(H+L)$ (Proteintech, 1:200), fluorescein (FITC)-conjugated AffiniPure donkey anti-mouse IgG $(\mathrm{H}+\mathrm{L})$ (Proteintech, 1:200). Images of anterior horns were captured under a fluorescence microscopy (Olympus, Melville, USA).

\subsection{Haematoxylin and eosin staining}

At $24 \mathrm{~h}$ after SCII, rats were anesthetized and euthanised for haematoxylin and eosin staining assay. After fixed with $4 \%$ paraformaldehyde, the $L_{4-6}$ spinal cord segments were stained with hematoxylin and eosin (H\&E) staining kit (Servicebio G1005, China). ${ }^{20}$ Morphology of Neurons in the stained areas was examined by two unfamiliar observers with an optical microscope (Leica, Germany). The occurrence of diffusely eosinophilic cytoplasm with pyknotic homogenous nuclei and basophilic stippling was utilized to identify the necrotic or normal neurons, respectively [21,22]. The number of normal motor neurons in the anterior horn was calculated as the average counts from three sections [23].

\subsection{Statistical analysis}

GraphPad Prism 7.0 (CA, USA) and SPSS 25.0 (CA, USA) were used to analyze the statistical results. Data were expressed as the mean \pm SD. Normally distributed data were performed by the t-tests, one-way or two-way ANOVA, while non-normally distributed data were performed by Kruskal-Wallis test. Results with a value of $p<0.05$ were regarded with statistical significance

\section{Results}

\subsection{Changes in behavioral assessment and MMP-7 expression after SCII}

As shown in Fig. 2A, behavioral assessment was performed by Tarlov criteria at $12 \mathrm{~h}$ intervals within $48 \mathrm{~h}$ after SCII. Compared with the sham group, the average Tarlov scores in SCII group were greatly decreased within $48 \mathrm{~h}$ after surgery $(\mathrm{p}<0.05)$. As shown in Fig. 2B-D, compared with the sham group, the expression of MMP-7 was noticeably upregulated in SCII $24 \mathrm{~h}$ group according to PCR and Western blot $(p<0.05)$. Based on the above findings, the SCII-24h time point was used to assess the effect of MMP-7.

\subsection{The expression and localization of MMP-7 protein after SCII}

The expression and localization of MMP-7 with GFAP, Iba-1 and NeuN was evaluated by Double immunofluorescence labeling (Fig. 3). As shown in Fig. 3A, red and green fluorescence represented MMP7 and markers of the main cell types (GFAP, Iba-1 and NeuN) respectively, and yellow fluorescence represented the co-localization. MMP-7 was mostly expressed in NeuN-positive neurons. Compared with the sham group, the fluorescent density of MMP-7 greatly increased in SCII-24h group (Fig. 3B, p < 0.05). 
As shown in Fig. 3C, the results demonstrated that SCll aggrandized the double-positive cell number of MMP-7 and NeuN ( $<<0.05)$.

\subsection{Silencing MMP-7 improved neurological deficits and histologic evaluation after SCII}

Lower limb motor function in each experimental group was evaluated by Tarlov criteria (Fig. 4A). Compared with the sham group, Tarlov scores in SCII group were greatly decreased $(p<0.05)$; compared with the SCII group, Tarlov scores in si-MMP7 group were greatly increased ( $<0.05)$, and Tarlov scores were similar in the NC group and SCII group ( $p>0.05)$. The data revealed that silencing MMP-7 could improve neurological deficits after SCII.

At $24 \mathrm{~h}$ after SCIl, histologic evaluation demonstrated that intact neurons in the anterior horn were greatly reduced, as shown for three signs: pyknotic homogenous nuclei, diffusely eosinophilic cytoplasm and basophilic stippling (Fig. 4B-C, p < 0.05). However, the si-MMP7 group demonstrated more intact neurons compared with the SCII group $(p<0.05)$. The data showed that histologic evaluation could be improved by silencing MMP-7 after SCII.

\subsection{Silencing MMP-7 decreased MMP-7 expression in neurons}

As shown in Fig. 5, MMP-7 was mostly expressed in NeuN-positive neurons. Compared with the sham group, fluorescence intensity of MMP-7 and number of co-localized cells in the SCll group was greatly increased ( $p<0.05$ ), while the quantitative analysis in si-MMP7 group was greatly decreased compared with the SCII group ( $p<0.05)$. It was similar in the NC group and SCII group $(p>0.05)$. It can be concluded that si-MMP7 could affect SCII by restraining MMP-7 expression in neurons.

\subsection{The protection by silencing MMP-7 against SCII through restraining the MMP-7/ cleaved caspase-3 axis and IL-1 $\beta$.}

As shown in Fig. 6A-D, compared with the sham group, the protein expression of MMP-7, IL-1 13 and cleaved caspase-3 in SCII group was greatly increased $(p<0.05)$, while the protein expression in si-MMP7 group was greatly reduced compared with the SCII group $(p<0.05)$. The protein expression was similar in the NC group and SCII group ( $p>0.05$ ). Meanwhile, the RNA expression of MMP-7 was also reduced by silencing MMP-7 after SCII (Fig. 6E, p < 0.05). As shown in Fig. 6F, MMP-7 fluorescence was co-localized with cleaved caspase-3 fluorescence, suggesting that MMP-7 might play partially roles by cleaved caspase-3. The changing trend of apoptotic factor and inflammatory cytokine was closely related to that of MMP-7 according to Fig. 6. The data showed that neuroapoptosis and inflammation could be restrained by silencing MMP-7 after SCII.

\section{Discussion}


We identified the role of MMP7 in SCll and studied the mechanism on neuroapoptosis and inflammation. MMP-7 expression was noticeably increased at the $24 \mathrm{~h}$ time point after surgery. Silencing MMP-7 showed a protective effect after SCII by improving lower limb motor function and histologic evaluation, along with reducing the expression of MMP-7, apoptotic factor and inflammatory cytokine. It was concluded that silencing MMP-7 protected the rats against SCII by inhibiting the neuroapoptosis and inflammation.

During nervous system development and following injury, MMPs have been increasingly recognized as playing vital roles [24]. For example, MMP-9 was associated with the early process of neuropathic pain in $\mathrm{CCl}$ rats [25]; and in peripheral nerve regeneration, MMP-7 promoted myelin sheath formation and Schwann cell migration [15]. As the smallest member of MMPs, MMP-7 plays vital roles in many physiological processes [26]. In bactericidal and anti-inflammatory roles, MMP7-deficient mice are strongly protected against an otherwise lethal dose of LPS [27]. In our previous study [17], we suggested silencing IncRNA Gas 5 protected against SCII in a rat-model by regulating MMP-7, but the specific mechanism of MMP-7 remained unclear. In this study, MMP-7 expression was greatly upregulated along with the reduction of Tarlov scores after SCII, suggesting a probable relation between MMP-7 and dyskinesia. Based on the $24 \mathrm{~h}$ time point after SCIl, cellular distribution of MMP-7 with GFAP, Iba-1 and NeuN was evaluated by Double immunofluorescence labeling. The results revealed that MMP-7 was mostly expressed in NeuN-positive neurons and the fluorescent density of MMP-7 greatly increased after SCII, suggesting that MMP-7 could play vital roles in SCII at least partially through neurons.

RNA interference technology is widely utilized to regulate the expression of individual genes and study the cellular function. In addition, siRNA technology was used to perform targeted treatment of protein genes and study the roles of specific proteins [28]. In our research, the protein and RNA expression of MMP-7 was greatly reduced by intrathecal injection of si-MMP7. Meanwhile, the IL-1 $\beta$ and cleaved caspase- 3 was greatly reduced by intrathecal injection of si-MMP7. IL-1 $\beta$ and cleaved caspase- 3 are common inflammatory cytokine and apoptotic factors, respectively [29]. Therefore, silencing MMP-7 could inhibit the neuroapoptosis and inflammation. In addition, silencing MMP-7 could improve lower limb motor function and histologic evaluation and reduce the expression of MMP-7 in neurons. Overall, silencing MMP-7 exerted neuroprotection on rat spinal cords against SCII.

In a study of MS lesions, double staining showed that MMP-7 was evidently localized to Iba1-positive microglia at 3 and 5 weeks demyelination [16], indicating that MMP-7 was closely related to microglia function. In our study, MMP-7 was mostly expressed in NeuN-positive neurons, indicating that MMP-7 was closely related to neurons function. It may be due to different disease models, tissue sites and timing. Additionally, the function of neurons and microglia may be partially in series. Of the major cell types of spinal cords, neurons are closely associated with lower limb motor function after SCII [4]. Therefore, MMP-7, which was mainly expressed in NeuN-positive neurons, could affect lower limb motor function after SCll at least partly through neurons. As shown in Fig. 6F, MMP-7 fluorescence was colocalized with cleaved caspase-3 fluorescence, suggesting that MMP-7 might play partially roles by cleaved caspase-3. Neuroapoptosis was now known to be the vital factor related to neuronal loss during 
SCII [30]. Damage from ischemia reperfusion could inhibit neuronal repair systems and induce caspasemediated neuroapoptosis [30, 31]. Therefore, MMP7, which was co-localized with NeuN and cleaved caspase-3 respectively, was closely related to the mechanism of neuroapoptosis induced by SCII. In addition, silencing MMP-7 could increase Tarlov scores and reduce the expression of MMP-7 in neurons and cleaved caspase-3 protein expression. Overall, silencing MMP-7 ameliorated SCII-induced neuroapoptosis through restraining the MMP-7/ cleaved caspase-3 axis.

This study will help researchers exploit potential therapeutic targets and diagnostic biomarkers for SCII. In the clinical treatment of MMP-7 after SCII, the detailed molecular mechanisms of MMP-7 need more additional research and cell experiments in vitro. The mechanism of neuroapoptosis has been elaborated, but more detailed mechanisms of inflammation or others require further study.

\section{Conclusions}

Collectively, we report that MMP-7 exerts vital function on SCII. Silencing MMP-7 protects the rats against SCIl by inhibiting the neuroapoptosis and inflammation. Silencing MMP-7 could be a hopeful therapeutic treatment after SCII.

\section{Abbreviations}

spinal cord ischemia-reperfusion injury: SCII; matrix metalloproteinase-7: MMP-7; Interleukin-1 $\beta$ : IL-1ß; polymerase chain reaction: PCR; negative control: NC; neuronal nuclei: NeuN; Matrix metalloproteinases: MMPs; small interfering RNAs: siRNAs; immunofluorescence: IF.

\section{Declarations}

\section{Acknowledgments:}

None

\section{Authors' contributions:}

Zailii Zhang conceived the idea and designed the experiments for the study. Dan Wang interpreted the data. Te Fang and Fengshou Chen performed the experiments. Te Fang wrote the manuscript. The authors read and approved the final manuscript.

\section{Competing interests:}

The authors declare that they have no competing interests

\section{Funding:}

None 
Availability of data and materials:

The data and materials used in the current study are all available from the corresponding author upon reasonable request.

\section{Ethics approval and consent to participate:}

The Ethics were approved by the Ethics Review Committee of China Medical University.

\section{Consent for publication:}

Not applicable.

\section{References}

1. Awad H, Ramadan ME, El Sayed HF, et al. Spinal cord injury after thoracic endovascular aortic aneurysm repair. Can J Anaesth 2017; 64(12):1218-1235.

2. Gkremoutis A, Schmandra T, Meyn M, et al. Hybrid approach to emergent and urgent treatment of complex thoracoabdominal aortic pathology. Eur J Vasc Endovasc Surg 2014; 48(4):407-13.

3. Preventza O, Cervera R, Cooley DA, et al. Acute type I aortic dissection: traditional versus hybrid repair with antegrade stent delivery to the descending thoracic aorta. J Thorac Cardiovasc Surg 2014; 148(1):119-25.

4. Li XQ, Yu Q, Tan WF, et al. MicroRNA-125b mimic inhibits ischemia reperfusion-induced neuroinflammation and aberrant p53 apoptotic signalling activation through targeting TP53INP1. Brain Behav Immun 2018; 74:154-165.

5. Li XQ, Yu Q, Zhang ZL, et al. MiR-187-3p mimic alleviates ischemia-reperfusion-induced pain hypersensitivity through inhibiting spinal P2X7R and subsequent mature IL-1 $\beta$ release in mice. Brain Behav Immun 2019; 79:91-101.

6. Wang H, Yu Q, Zhang ZL, et al. Involvement of the miR-137-3p/CAPN-2 Interaction in IschemiaReperfusion-Induced Neuronal Apoptosis through Modulation of p35 Cleavage and Subsequent Caspase-8 Overactivation. Oxid Med Cell Longev 2020; 2020:2616871.

7. Verma RP, Hansch C. Matrix metalloproteinases (MMPs): chemical-biological functions and (Q) SARs. Bioorg Med Chem 2007; 15(6):2223-68.

8. Nguyen TT, Shynlova O, Lye SJ. Matrix Metalloproteinase Expression in the Rat Myometrium During Pregnancy, Term Labor, and Postpartum. Biol Reprod 2016; 95: 24.

9. Ke B, Fan C, Yang L, et al. Matrix metalloproteinases-7 and kidney fibrosis. Front Physiol 2017; 8:21.

10. Tan RJ, Liu Y. Matrix metalloproteinases in kidney homeostasis and diseases. Am J Physiol Renal Physiol 2012; 302:F1351-F1361.

11. Yu B, Liu X, Chang H. MicroRNA-143 inhibits colorectal cancer cell proliferation by targeting MMP7. Minerva Med 2017 Feb; 108(1):13-19. 
12. Song $\mathrm{N}$, Liu $\mathrm{H}, \mathrm{Ma} X$, et al. Placental growth factor promotes metastases of ovarian cancer through MiR-543-regulated MMP7. Cell Physiol Biochem 2015; 37(3):1104-12.

13. Fu $H$, Zhou $D$, Zhu $H$, et al. Matrix metalloproteinase-7 protects against acute kidney injury by priming renal tubules for survival and regeneration. Kidney Int 2019; 95(5):1167-1180.

14. Qin J, Zha GB, Yu J, et al. Differential temporal expression of matrix metalloproteinases following sciatic nerve crush. Neural Regen Res 2016; 11(7):1165-71.

15. Wang $\mathrm{H}$, Zhang $\mathrm{P}, \mathrm{Yu}$ J, et al. Matrix metalloproteinase 7 promoted Schwann cell migration and myelination after rat sciatic nerve injury. Mol Brain 2019; 12(1):101.

16. Wang P, Gorter RP, de Jonge JC, et al. MMP7 cleaves remyelination-impairing fibronectin aggregates and its expression is reduced in chronic multiple sclerosis lesions. Glia 2018; 66: 1625-1643.

17. Zhang Z, Li X, Chen F, et al. Downregulation of LncRNA Gas5 inhibits apoptosis and inflammation after spinal cord ischemia-reperfusion in rats. Brain Res Bull. 2021; 168:110-119.

18. Li XQ, Yu Q, Chen FS, et al. Inhibiting aberrant p53-PUMA feedback loop activation attenuates ischaemia reperfusion-induced neuroapoptosis and neuroinflammation in rats by downregulating caspase 3 and the NF-KB cytokine pathway. J Neuroinflammation 2018; 15(1):250.

19. Jia H, Ma H, Li Z, et al. Downregulation of LncRNA TUG1 Inhibited TLR4 Signaling PathwayMediated Inflammatory Damage After Spinal Cord Ischemia Reperfusion in Rats via Suppressing TRIL Expression. J Neuropathol Exp Neurol 2019; 78(3):268-282

20. Wang D, Chen F, Fang B, et al. MiR-128-3p Alleviates Spinal Cord Ischemia/Reperfusion Injury Associated Neuroinflammation and Cellular Apoptosis via SP1 Suppression in Rat. Front Neurosci 2020; 14:609613.

21. Guan C, Luan L, Li J, Yang L. MiR-212-3p improves rat functional recovery and inhibits neurocyte apoptosis in spinal cord injury models via PTEN downregulation-mediated activation of AKT/mTOR pathway. Brain Res. 2021;1768:147576.

22. Chen F, Li X, Li Z, et al. The roles of chemokine (C-X-C motif) ligand 13 in spinal cord ischemiareperfusion injury in rats. Brain Res 2020; 1727:146489.

23. Fang B, Li XQ, Bi B, et al. Dexmedetomidine attenuates blood-spinal cord barrier disruption induced by spinal cord ischemia reperfusion injury in rats. Cell Physiol Biochem 2015; 36(1):373-383.

24. Yong VW. Metalloproteinases: mediators of pathology and regeneration in the CNS. Nat Rev Neurosci 2005; 6(12):931-944.

25. Zhao L, Song C, Huang Y, et al. MMP-9 regulates CX3CL1/CX3CR1 in the early phase of neuropathic pain in chronic sciatic nerve constriction injury (CCI) rats. Ann Palliat Med 2020; 9(4):2020-2027.

26. Dejonckheere $\mathrm{E}$, Vandenbroucke RE, Libert C. Matrix metalloproteinases as drug targets in ischemia/reperfusion injury. Drug Discov Today 2011; 16(17-18):762-778.

27. Vandenbroucke RE, Vanlaere I, Van Hauwermeiren F, et al. Pro-inflammatory effects of matrix metalloproteinase 7 in acute inflammation. Mucosal Immunol 2014; 7(3):579-588.

28. Han H. RNA Interference to Knock Down Gene Expression. Methods Mol Biol 2018; 1706:293-302. 
29. Chen M, Gao YT, Li WX, et al. FBW7 protects against spinal cord injury by mitigating inflammationassociated neuronal apoptosis in mice. Biochem Biophys Res Commun 2020; 532(4):576-583.

30. Li XQ, Cao XZ, Wang J, et al. Sevoflurane preconditioning ameliorates neuronal deficits by inhibiting microglial MMP-9 expression after spinal cord ischemia/reperfusion in rats. Mol Brain 2014; 7:69.

31. Huang YN, Yang LY, Greig NH, Wang YC, Lai CC, Wang JY. Neuroprotective effects of pifithrin-a against traumatic brain injury in the striatum through suppression of neuroinflammation, oxidative stress, autophagy, and apoptosis. Sci Rep. 2018;8(1):2368.

\section{Figures}

A Neurological function evaluation and expression of MMP-7 at different time points after SCII

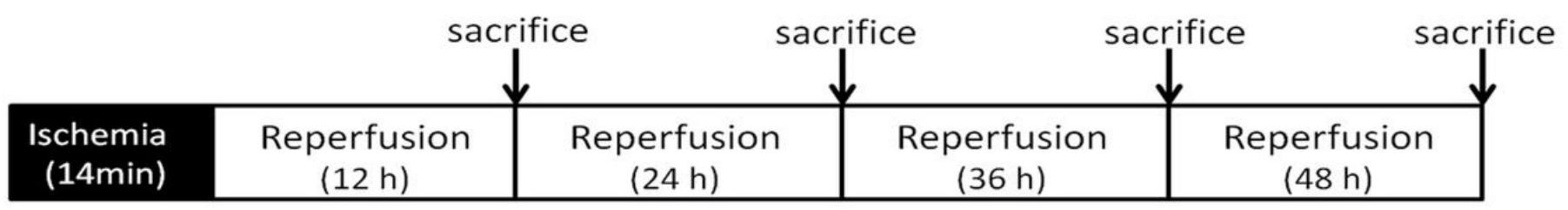

B Neurological function evaluation, qRT-PCT, Western blotting, HE and IF

\begin{tabular}{|c|c|c|}
\hline $25 \mu \mathrm{l}$ saline $3 \mathrm{~d}$ & Without ischemia $14 \mathrm{~min}$ & $\begin{array}{c}\text { Reperfusion } \\
(24 \mathrm{~h})\end{array}$ \\
\hline
\end{tabular}

\begin{tabular}{|ccc|c|}
\hline $25 \mu \mathrm{l}$ & saline $\quad 3 \mathrm{~d}$ & Ischemia $14 \mathrm{~min}$ & $\begin{array}{c}\text { Reperfusion } \\
(24 \mathrm{~h})\end{array}$ \\
\hline $25 \mu \mathrm{l}$ & $\mathrm{NC} \quad 3 \mathrm{~d}$ & Ischemia $14 \mathrm{~min}$ & $\begin{array}{c}\text { Reperfusion } \\
(24 \mathrm{~h})\end{array}$ \\
\hline & & & \\
\hline $25 \mu \mathrm{l}$ & si-MMP7 3d & Ischemia 14min & $\begin{array}{c}\text { Reperfusion } \\
(24 \mathrm{~h})\end{array}$ \\
\hline
\end{tabular}

\section{Figure 1}

Experimental protocol. (A) Neurological function evaluation and expression of MMP-7 at $12 \mathrm{~h}$ intervals within $48 \mathrm{~h}$ after SCII. (B) Neurological function evaluation, qRT-PCR, Western blot, HE and IF during four groups. 
A

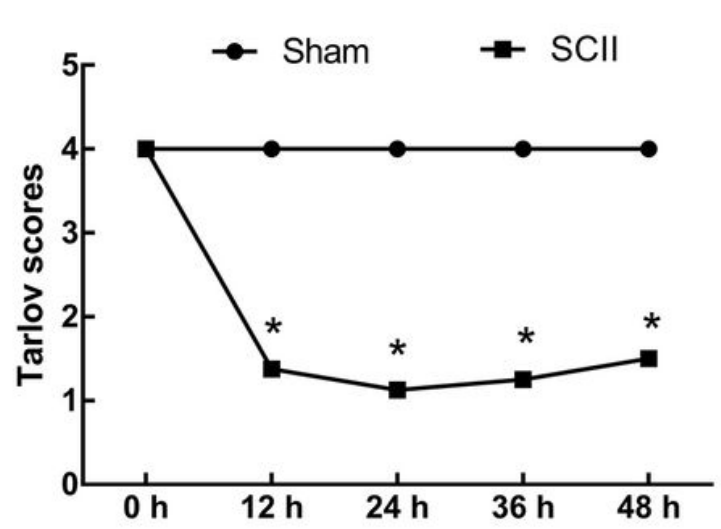

B

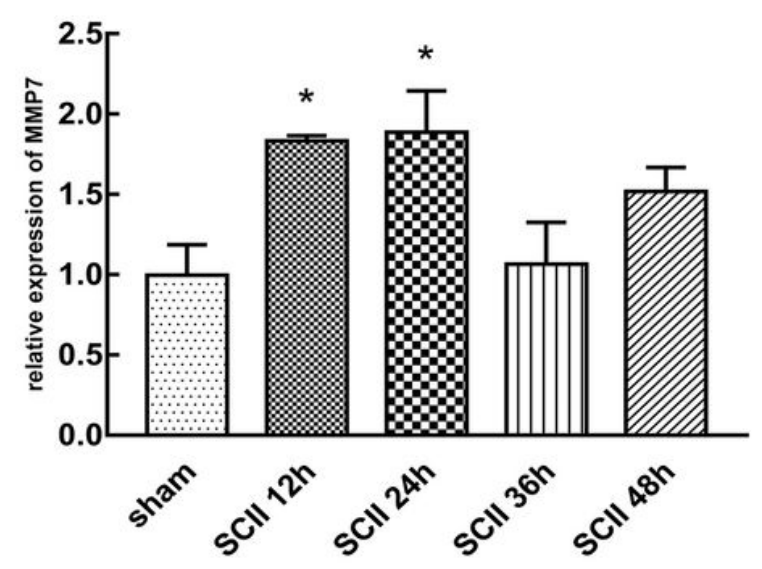

C

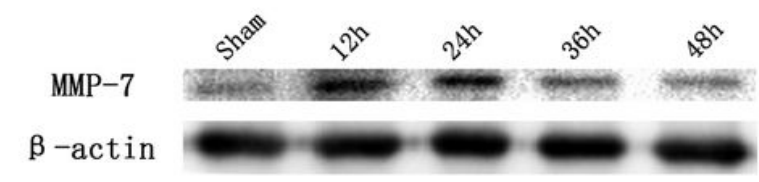

D

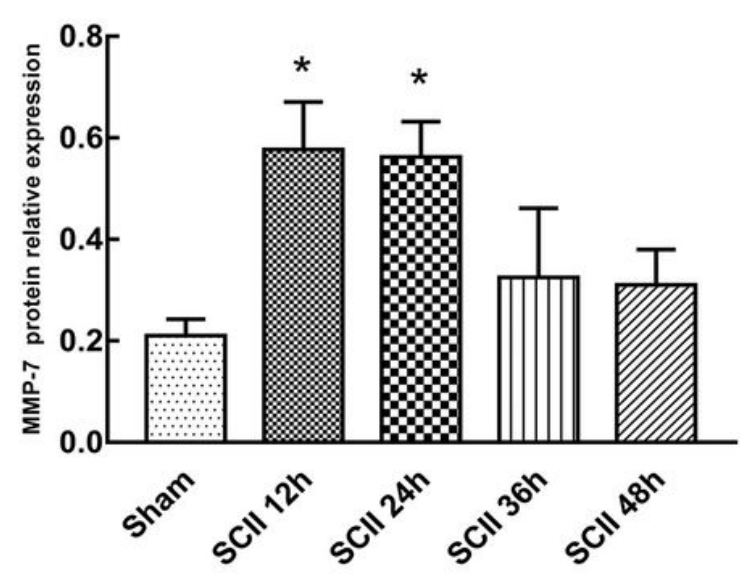

Figure 2

Changes in behavioral assessment and MMP-7 expression after SCII. (A) Neurological function was evaluated by Tarlov criteria at $12 \mathrm{~h}$ intervals within $48 \mathrm{~h}$ after SCII $(\mathrm{n}=8)$. (B) Relative MMP-7 mRNA expression at $12 \mathrm{~h}$ intervals within $48 \mathrm{~h}$ after SCII $(\mathrm{n}=4)$. (C-D) Relative MMP-7 protein expression at $12 \mathrm{~h}$ intervals within $48 \mathrm{~h}$ after SCII $(n=4)$. Data were expressed as the mean $\pm S D$. ${ }^{\star} p<0.05$, vs. sham group. 
A

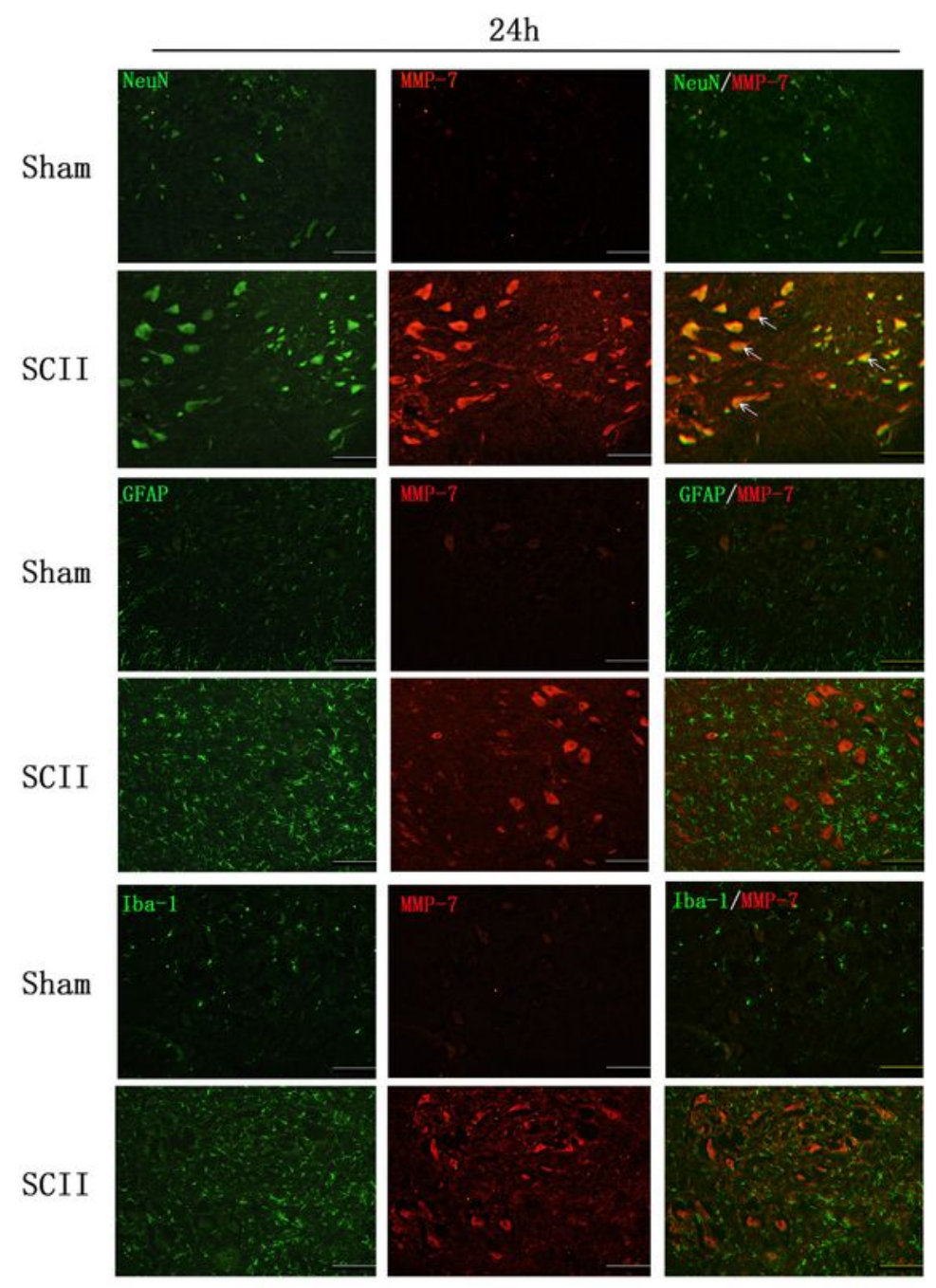

B

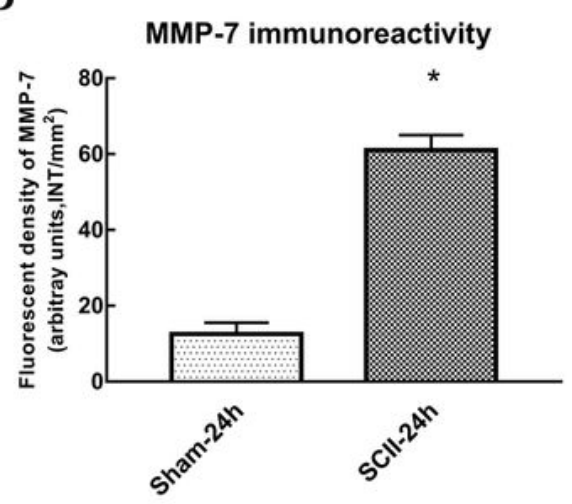

$\mathrm{C}$

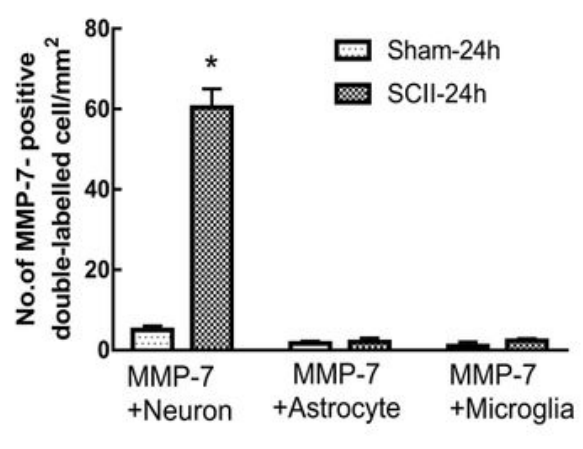

\section{Figure 3}

Cellular distribution of MMP-7 expression after SCII. (A) Representative images showed the colocalization of MMP-7 (red) with GFAP (green), Iba-1 (green) and NeuN (green). Co-localization was represented by yellow signals (white arrow). Scale bar: $100 \mu \mathrm{m}$. (B) Quantitative data were presented for fluorescent density of MMP-7 ( $n=3)$. (C) Quantitative data were presented for double-labeled cells $(n=3)$. Data were expressed as the mean $\pm S D$. ${ }^{*} p<0.05$, vs. sham group. 
A B
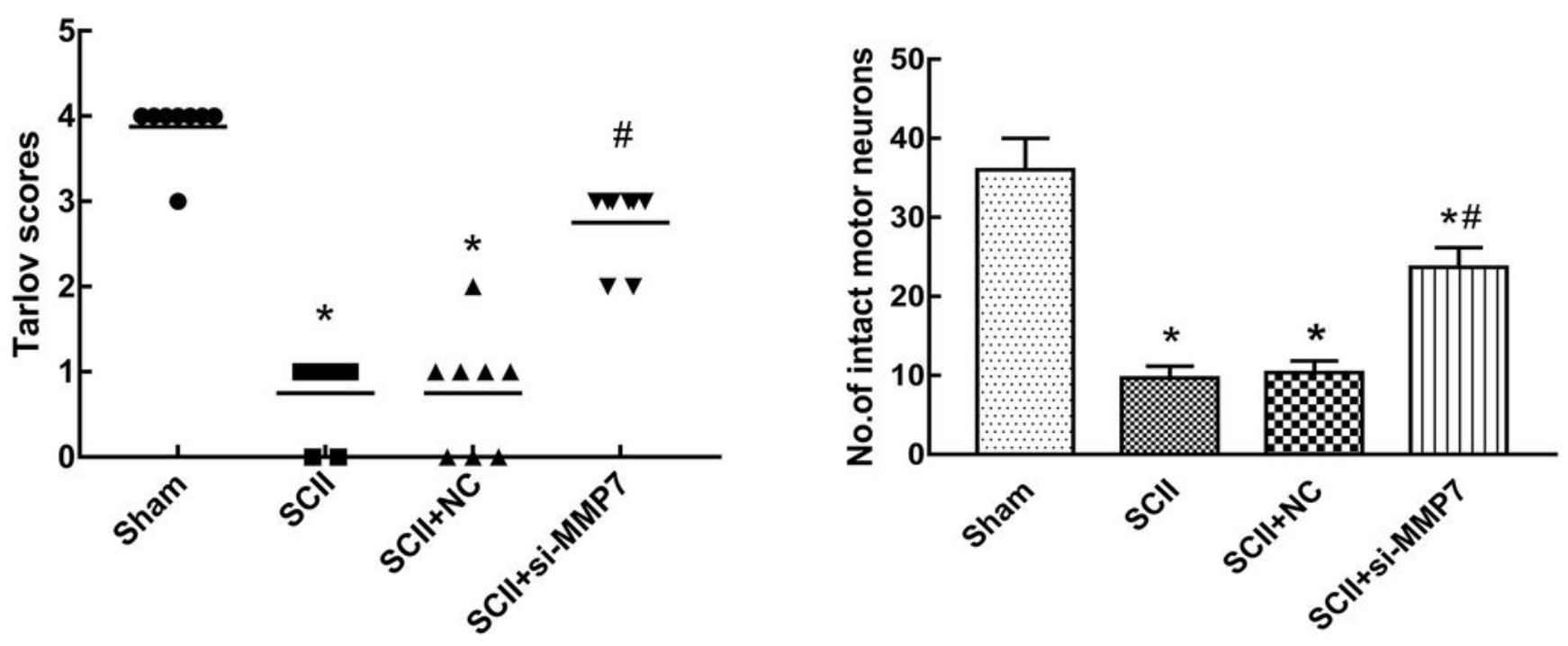

$\mathrm{C}$

Sham

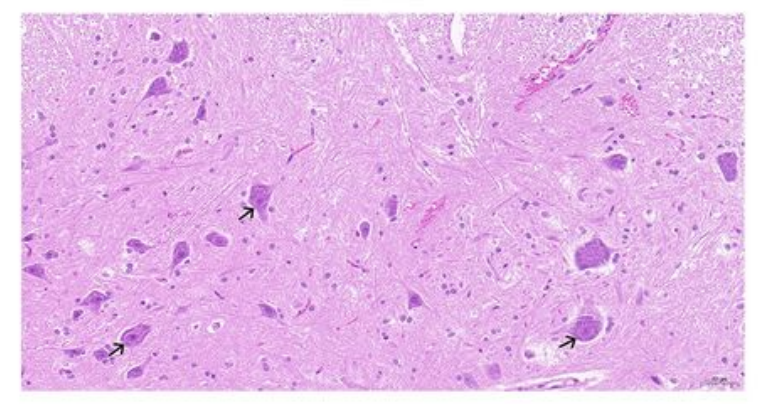

SCII

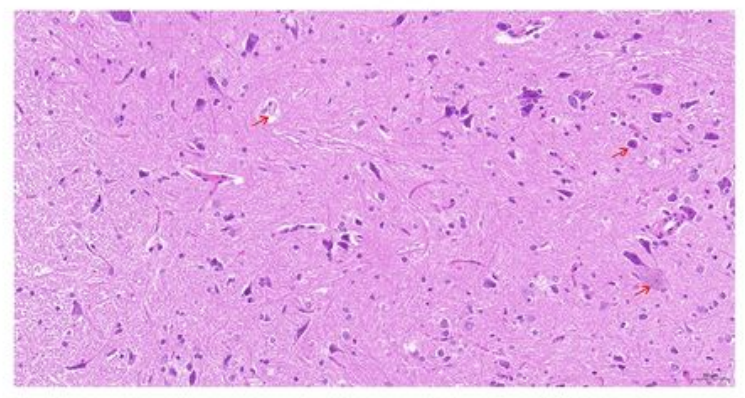

\section{$\mathrm{SCII}+\mathrm{NC}$}

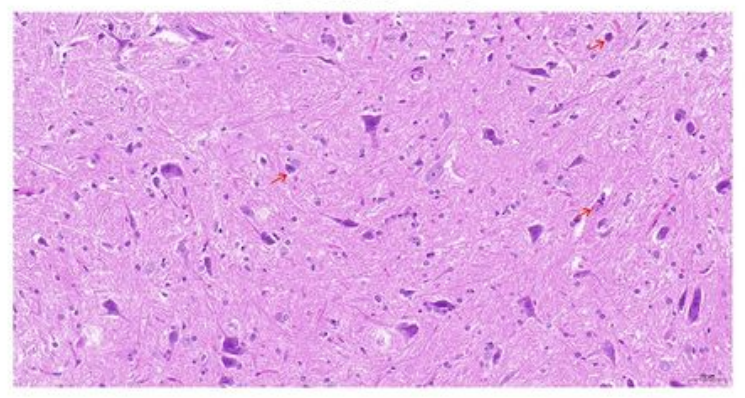

\section{SCII+si-MMP7}

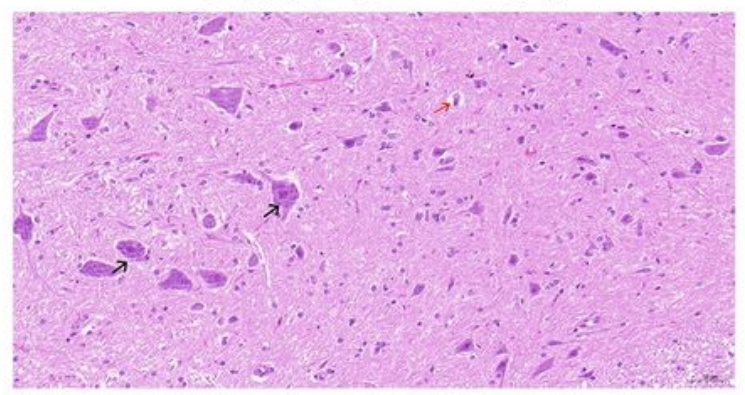

\section{Figure 4}

Lower limb motor function and histologic evaluation after SCII. (A) Lower limb motor function in each experimental group was evaluated by Tarlov criteria. Each rat was represented by a different sign $(n=8)$. $(B-C)$ The intact neurons in the anterior horn were demonstrated by histologic evaluation $(n=3)$. Scale bar: $50 \mu \mathrm{m}$. Data were expressed as the mean $\pm S D$. ${ }^{\star} p<0.05$, vs. sham group; $\# P<0.05$, vs. SCll group. 
A

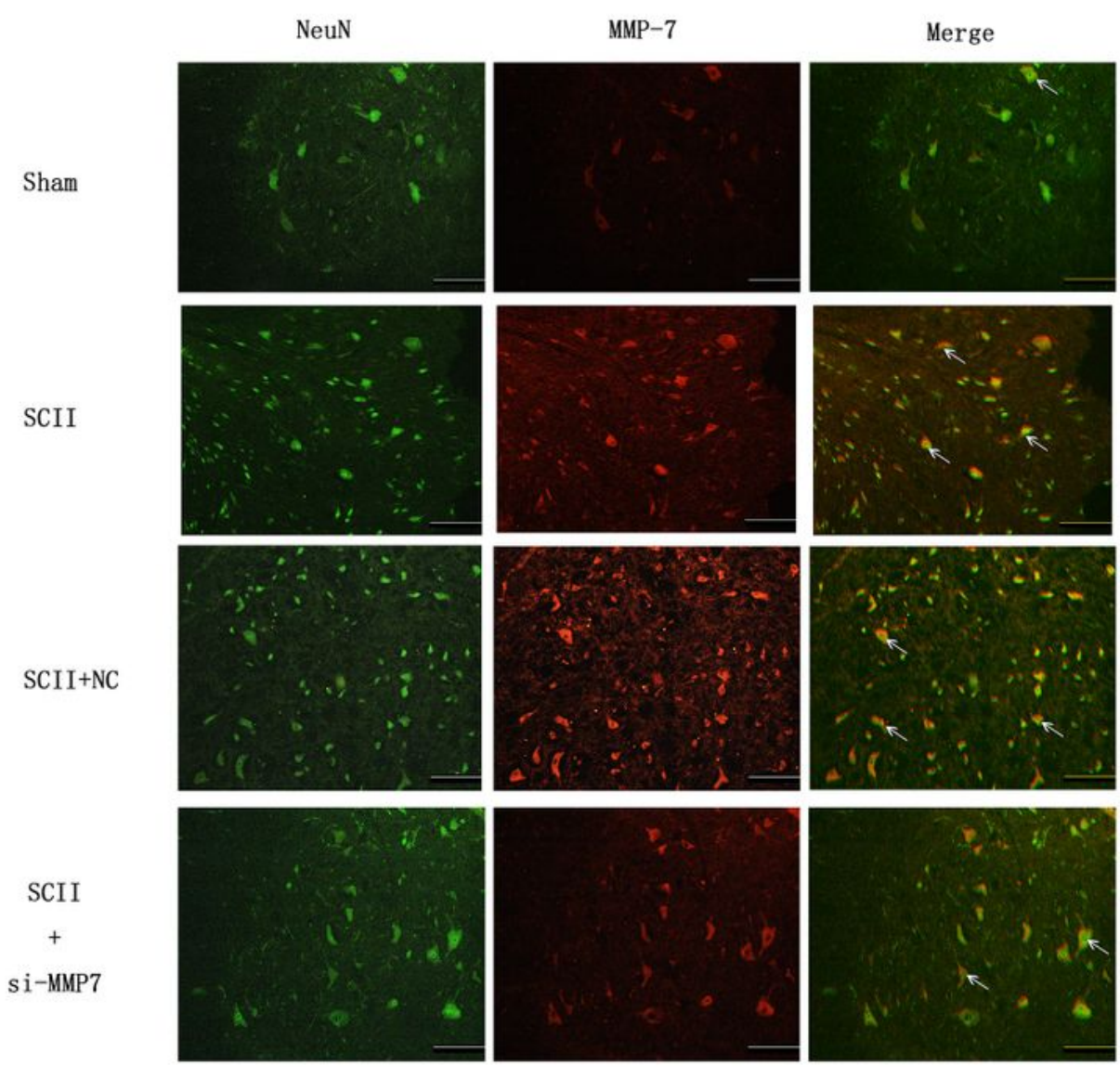

B

$\mathrm{C}$
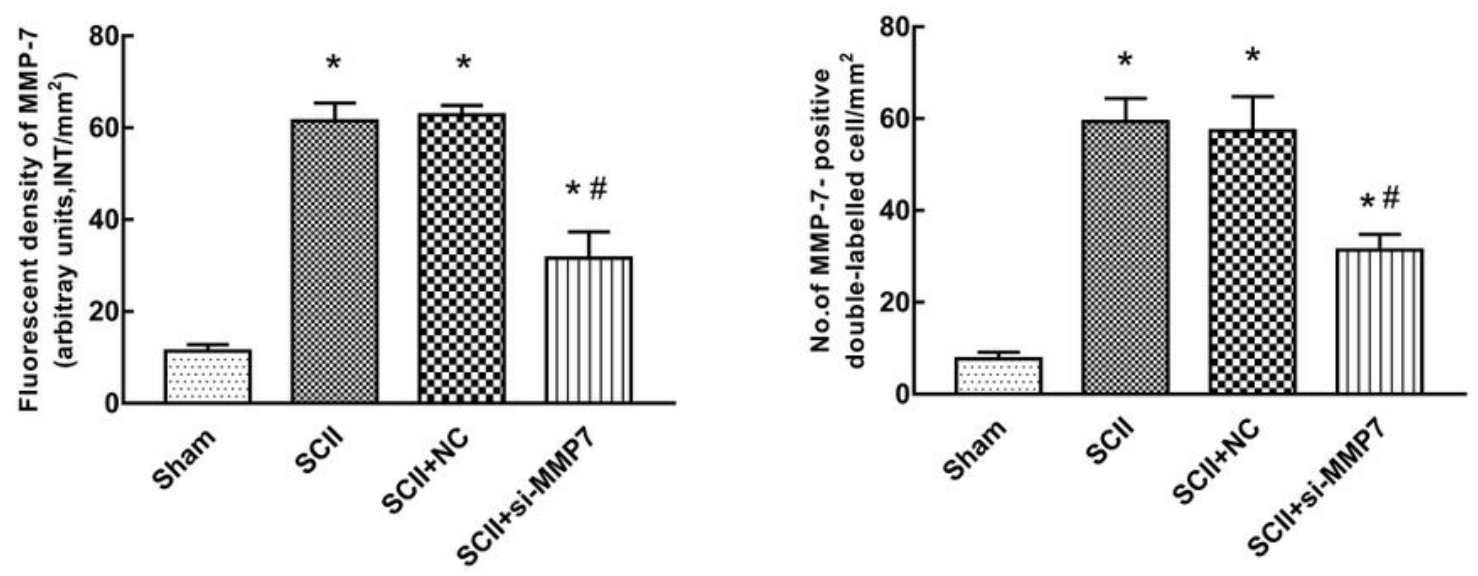

\section{Figure 5}

Fluorescent expression of MMP-7 in neurons after silencing MMP-7. (A) Representative images showed the co-localization of MMP-7 (red) with NeuN (green). Co-localization was represented by yellow signals (white arrow). Scale bar: $100 \mu \mathrm{m}$. MMP-7 was mostly expressed in NeuN-positive neurons. (B) Quantitative data were presented for fluorescent density of MMP-7 $(n=3)$. Silencing MMP-7 decreased MMP-7 fluorescent density. (C) Quantitative data were presented for double-labeled cells ( $n=3)$. Silencing 
MMP-7 decreased the number of co-localized cells. Data were expressed as the mean $\pm S D$. ${ }^{*} p<0.05$, vs. sham group; $\# P<0.05$, vs. SCII group.

A

MMP-7

Cleavd casepase-3

IL-1 $\beta$

$\beta$-actin

B

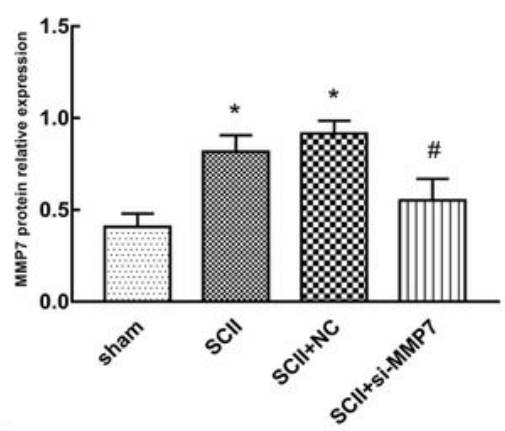

D

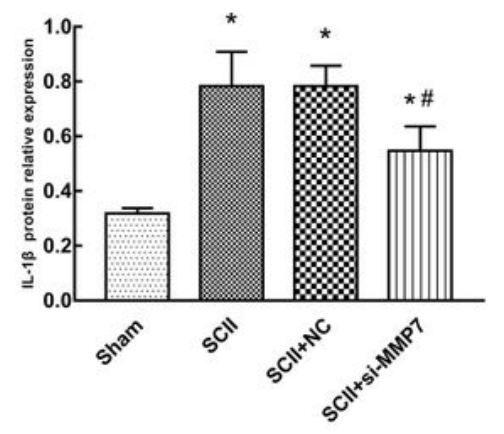

$\mathrm{F}$

MMP-7

Cleavd casepase- 3
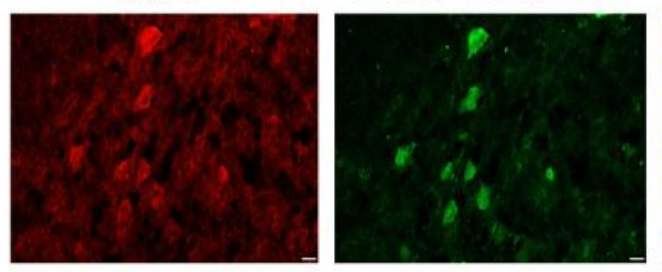

$\mathrm{C}$

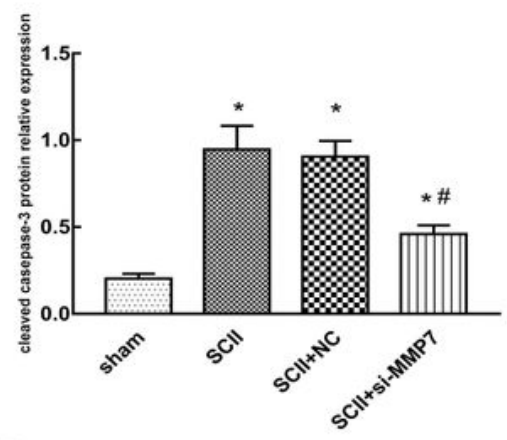

$\mathrm{E}$
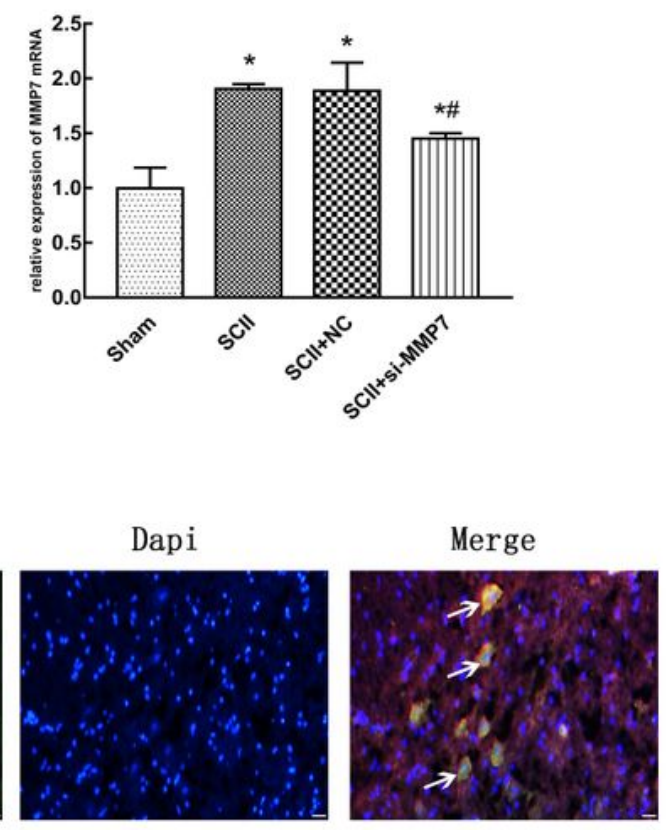

Figure 6

(A-D) Relative MMP-7, IL-1 $\beta$ and cleaved caspase-3 protein expression after silencing MMP-7 $(n=4)$. (E) Relative MMP-7 mRNA expression after silencing MMP-7 $(n=4)$. (F) Representative images showed the co-localization of MMP-7 (red) and cleaved caspase-3 (green). Co-localization was represented by yellow 
signals (white arrow). Scale bar: $20 \mu \mathrm{m}$. Data were expressed as the mean $\pm \mathrm{SD}$. ${ }^{\star} \mathrm{p}<0.05$, vs. sham group; $\# \mathrm{P}<0.05$, vs. SCII group. 